University of Wollongong

Research Online

Faculty of Social Sciences - Papers (Archive) Faculty of Arts, Social Sciences \& Humanities

2013

Can event-related potentials serve as neural markers for wins, losses, and near-wins in a gambling task? A principal components analysis

Lisa Lole

University of Wollongong, lisawood@uow.edu.au

Craig J. Gonsalvez

University of Wollongong, craigg@uow.edu.au

Robert J. Barry

University of Wollongong, rbarry@uow.edu.au

Frances M. De Blasio

University of Wollongong, francesd@uow.edu.au

Follow this and additional works at: https://ro.uow.edu.au/sspapers

Part of the Education Commons, and the Social and Behavioral Sciences Commons

Research Online is the open access institutional repository for the University of Wollongong. For further information contact the UOW Library: research-pubs@uow.edu.au 


\title{
Can event-related potentials serve as neural markers for wins, losses, and near- wins in a gambling task? A principal components analysis
}

\author{
Abstract \\ Originally, the feedback related negativity (FRN) event-related potential (ERP) component was considered \\ to be a robust neural correlate of non-reward/punishment processing, with greater negative deflections \\ observed following unfavourable outcomes. More recently, it has been suggested that this component is \\ better conceptualised as a positive deflection following rewarding outcomes. The current study sought to \\ elucidate the nature of the FRN, as well as another component associated with incentive-value \\ processing, the $\mathrm{P} 3 \mathrm{~b}$, through application of a spatiotemporal principal components analysis (PCA). \\ Seventeen healthy controls played a computer electronic gaming machine (EGM) task and received \\ feedback on credits won or lost on each trial, and ERPs were recorded. The distribution of reward/non- \\ reward outcomes closely matched that of a real EGM, with frequent losses, and infrequent wins and near- \\ wins. The PCA revealed that feedback elicited both a frontally maximal negative deflection to losses, and \\ a positive deflection to wins (which was also sensitive to reward magnitude), implying that the neural \\ generator/s of the FRN are differentially activated following these outcomes. As expected, greater P3b \\ amplitudes were found for wins compared to losses. Interestingly, near-wins elicited significantly smaller \\ FRN amplitudes than losses (with no differences in P3b amplitude), and may contribute to the \\ maintenance of gambling behaviours on EGMs. The results of the current study are integrated into a \\ response profile of healthy controls to outcomes of varying incentive value. This may provide a \\ foundation for the future examination of individuals who exhibit abnormalities in reward/punishment \\ processing, such as problem gamblers.
}

\section{Keywords}

event, can, principal, analysis, task, components, gambling, near, losses, wins, markers, neural, serve, potentials, related

\section{Disciplines}

Education | Social and Behavioral Sciences

\section{Publication Details}

Lole, L., Gonsalvez, C. J., Barry, R. J. \& De Blasio, F. M. (2013). Can event-related potentials serve as neural markers for wins, losses, and near-wins in a gambling task? A principal components analysis.

International Journal of Psychophysiology, 89 (3), 390-398. 


\section{Title Page}

Can event-related potentials serve as neural markers for wins, losses, and near-wins in a gambling task? A principal components analysis

Lisa Lole*, a, b $^{\text {, Craig J. Gonsalvez }}{ }^{\mathrm{a}, \mathrm{b}, \mathrm{c}}$, Robert J. Barry ${ }^{\mathrm{a}, \mathrm{b}}$, and Frances M. De Blasio ${ }^{\mathrm{a}, \mathrm{b}}$

a. School of Psychology, University of Wollongong, NSW 2522, Australia.

b. Brain \& Behaviour Research Institute, University of Wollongong, NSW 2522, Australia.

c. Illawarra Institute for Mental Health, University of Wollongong, NSW 2522, Australia.

*Corresponding author:

Phone: +61 24221 4513, fax: +61 24221 4163, email: lisawood@uow.edu.au

KEYWORDS: ERP; Electronic gaming machine; FRN; P300; Reward sensitivity; punishment sensitivity; principal components analysis (PCA)

WORD COUNT: Abstract: 246; Article body: 5351; Figures: 5; Tables: zero; Supplemental information: zero. 


\begin{abstract}
Originally, the feedback related negativity (FRN) event-related potential (ERP) component was considered to be a robust neural correlate of non-reward/punishment processing, with greater negative deflections observed following unfavourable outcomes. More recently, it has been suggested that this component is better conceptualised as a positive deflection following rewarding outcomes. The current study sought to elucidate the nature of the FRN, as well as another component associated with incentive-value processing, the $\mathrm{P} 3 \mathrm{~b}$, through application of a spatiotemporal principal components analysis (PCA). Seventeen healthy controls played a computer electronic gaming machine (EGM) task and received feedback on credits won or lost on each trial, and ERPs were recorded. The distribution of reward/non-reward outcomes closely matched that of a real EGM, with frequent losses, and infrequent wins and near-wins. The PCA revealed that feedback elicited both a frontally maximal negative deflection to losses, and a positive deflection to wins (which was also sensitive to reward magnitude), implying that the neural generator/s of the FRN are differentially activated following these outcomes. As expected, greater P3b amplitudes were found for wins compared to losses. Interestingly, near-wins elicited significantly smaller FRN amplitudes than losses (with no differences in P3b amplitude), and may contribute to the maintenance of gambling behaviours on EGMs. The results of the current study are integrated into a response profile of healthy controls to outcomes of varying incentive value. This may provide a foundation for the future examination of individuals who exhibit abnormalities in reward/punishment processing, such as problem gamblers.
\end{abstract}




\section{Can event-related potentials serve as neural markers for wins, losses, and near-wins in a gambling task? A principal components analysis}

\section{Introduction}

The neural mechanisms involved in the processing of reward and non-reward/punishment are of particular relevance to addictive disorders, such as problem gambling, as abnormalities in incentive value processing are believed to be one of the causal factors in such disorders. For example, problem gamblers may be hyposensitive to non-reward/punishment (e.g., Reuter et al., 2005) and thus, the repeated detrimental losses experienced are not perceived to be averse; they may be hypersensitive to reward (e.g., Hewig et al., 2010; Oberg et al., 2011) and pursue wins at the expense of high costs; or they may be hyposensitive to reward (e.g., Blum et al., 2000) and engage in thrill-seeking behaviour (such as trying to obtain large wins) in order to reach the same level of excitement associated with smaller wins in non-problem gamblers.

A particularly valuable index of incentive value processing is an event-related potential (ERP) component; the feedback related negativity (FRN), as it has been demonstrated to be a robust and reliable ERP component sensitive to valence manipulations. The FRN is maximal at fronto-central scalp sites and there is consensus that medial frontal cortical areas, especially the anterior cingulate cortex (ACC), are involved in its generation (Bellebaum and Daum, 2008; Miltner et al., 1997; Nieuwenhuis et al., 2004). Because of potential links to reward mechanisms through activation of the mesencephalic dopamine system (Holroyd and Coles, 2002), the FRN has major significance, particularly for gambling behaviours, as it provides a window through which the effects of reward and non-reward outcomes within the brain might be usefully examined. 
Recently, there has been debate regarding the nature of this ERP component; specifically, whether it is best conceptualised as a negative deflection following unfavourable outcomes or as a positive deflection following favourable outcomes. Earlier conceptualisations of the FRN were that it is a component characterised by greater negative responses $250-350 \mathrm{~ms}$ following feedback that signals monetary losses compared to gains (San Martin et al., 2010; Toyomaki and Murohashi, 2005; Yeung et al., 2005), or the least desired of two possible outcomes within a certain context (e.g., zero credits elicited larger FRNs than wins when the alternative outcome is to gain credits, compared to when the alternative is to lose credits; Gehring and Willoughby, 2002; Holroyd et al., 2004), during tasks that resemble gambling activity. Subjective expectancy of an outcome has also been shown to affect the FRN, with larger amplitudes associated with unexpected compared to predicted negative outcomes, although this effect appears to be more subtle and may not always follow objective probabilities of such events (Hajcak et al., 2005, 2006). Whilst the link between FRN and valence appears consistent, manipulations of incentive value magnitude have yielded equivocal results. Specifically, some studies suggest that larger losses (compared to smaller losses) and smaller gains (compared to larger gains) yield larger FRN magnitudes (e.g., Bellbaum et al., 2010; Holroyd et al., 2004), whilst others have found no magnitude effects (e.g., Gu et al., 2010; Hajcak et al., 2006; Yeung and Sanfey, 2004).

While the negative deflection to unfavourable outcomes described above has been reported in a wide variety of circumstances, including simulated gambling (Hewig et al., 2007), guessing tasks (Hajcak et al., 2006; Hajcak et al., 2005, 2007), time estimation tasks (Holroyd and Krigolsen, 2007; Miltner et al., 1997; Nieuwenhuis et al., 2005), and learning tasks (De Pascalis et al., 2010), the true nature of the FRN remains somewhat unclear, as this component is commonly superimposed on large amplitude P300 responses that occur immediately after it. It has been proposed that the reduced amplitude FRN observed 
following win outcomes may not be an actual attenuated response to these events, but is rather driven by larger P300 amplitudes following favourable outcomes (Yeung and Sanfey, 2004). Furthermore, the relative contribution of negative and positive outcomes to the FRN remains unclear due to the fact that many studies have employed the computation of a difference waveform to measure FRN magnitude (e.g., Dunning and Hajcak, 2007; Foti and Hajcak, 2009; Hajcak et al., 2007; Holroyd et al., 2008; Miltner et al., 1997), and other recent research has suggested that, rather than a negative deflection to non-reward outcomes, the FRN is better conceptualised as a positive deflection that is greater following reward compared to non-reward outcomes (Foti et al., 2011; Holroyd et al., 2003; Holroyd et al., 2008).

Regardless of the actual response pattern, investigation of the latent spatial and temporal characteristics of this feedback related ERP component (whether it be a negative deflection to non-reward or a positive deflection to reward) in healthy controls using a principal components analysis (PCA) will allow a more reliable and accurate account of the neural correlates associated with incentive value processing. This will encourage the future examination of whether these responses differ in individuals who display deficits in outcome evaluation, such as those with gambling problems.

Typically examined as a global component, the P300 (called the LPC in many studies), has also been shown to be sensitive to various aspects of incentive value on tasks that simulate gambling (Bellbaum et al., 2010; Hajcak et al., 2007). The inverse relationship between probability and P300 amplitude has been well established (Donchin and Coles, 1988), although the understanding of these results is subject to different interpretations (see Gonsalvez et al., 2007; Verleger, 1988). Nevertheless, studies that have controlled for event probability have demonstrated that the $\mathrm{P} 300$ remains sensitive to manipulations of win and loss outcomes (e.g., Hajcak et al., 2007; Wu and Zhou, 2009; Yeung et al., 2005; Zhou et al., 
2010), although the pattern of these results is somewhat variable. Some studies report a double dissociation between the FRN and P300, showing the FRN to be affected by valence but not reward magnitude, with the opposite pattern for the P300, regardless of whether the outcome is of positive or negative valence (Sato et al., 2005; Yeung and Sanfey, 2004). In contrast to this, other research has demonstrated that the P300 is influenced by valence, with wins eliciting larger amplitudes than losses (Hajack et al., 2007; Toyomaki and Murohashi, 2005). Because the P300 is established to be a complex comprising several sub-components, it is possible that different subcomponents are independently sensitive to valence and magnitude. For instance, stimulus salience is known to affect the P3b and win events may elicit larger P3bs on account of their greater salience than losses. Therefore, it is of value to determine which of the sub-components of the P300 are affected by win and loss outcomes. By using a spatiotemporal PCA, the current study sought to examine the latent nature of both the FRN and the LPC ERP subcomponents, that may not be perceptible using traditional ERP data extraction methods, in response to manipulations of valence and magnitude within a simulated electronic gaming machine (EGM; also called a 'poker' or 'slot' machine) task. EGMs typically deliver a large number of win and loss outcomes in a short period of time and are of particular clinical significance to problem gambling. Compared with other gambling activities, a high percentage of gamblers seeking treatment report addiction to EGMs (see Dowling et al., 2001), and EGM gambling is associated with a faster progression of addiction (Breen and Zimmerman, 2002) and more severe symptoms (Petry, 2003). In the current study all key EGM outcomes were of interest, including large and small wins, losses, and near-wins (see Method section 2.2.2. for details on these outcomes). Traditional ERP research has shown near wins to be less aversive (Luo et al., 2011) and more rewarding than losses (Qi et al., 2011), and neuroimaging research has shown that, while these outcomes are rated as more unpleasant than losses, they increase motivation to gamble by recruiting reward 
related brain circuitry (Clark et al., 2009). The current study sought to examine whether the latent neural correlates of incentive value processing are differentially activated for these outcomes compared to losses, in order to evaluate their role in the development and maintenance of gambling behaviours.

In summary, the current study sought to utlilise a PCA to parse two ERP components previously found to index various aspects of incentive value processing from overlapping data, and to evaluate their capacity to discriminate between win, loss, and near-win outcomes, as well as rewards of different magnitudes.

\section{Method}

\subsection{Participants}

Seventeen undergraduate psychology students ( 7 male, 10 female, $M_{\text {age }}=18.7$ years; $S D=4.8$, age range $=18-23$ years) from the University of Wollongong participated in the experiment in return for course credit. No participants reported using nicotine, alcohol, or prescription/illicit drugs in the two hours prior to testing, or a history of severe brain injury or seizures. Written informed consent was obtained from all participants, who were advised that participation was entirely voluntary, and that they could withdraw from the study at any time. The study's protocol was approved by the University of Wollongong Human Research Ethics Committee.

\subsection{Materials}

\subsubsection{Recording equipment}

EEG was recorded from a 19-site electrode cap (comprised of tin electrodes fitted in the standard international 10-20 system layout) using NuAmps 2.0 software (NeuroScan 
Compumedics, USA). The electrodes were referenced to linked ears and grounded by a cap electrode located mid-way between Fpz and Fz. Vertical eye movement (vEOG) was monitored with two tin cup electrodes: one placed $2 \mathrm{~cm}$ above and the other $2 \mathrm{~cm}$ below the left eye. Horizontal eye movement (hEOG) was monitored with two tin cup electrodes placed adjacent to the outer canthus of each eye. Impedance was less than $5 \mathrm{k} \Omega$ for cap electrodes and less than $3 \mathrm{k} \Omega$ for EOG and reference electrodes. Scalp EEG potentials were amplified $\times$ 20000 , EOG potentials were amplified $\times 5000$, and both were sampled at a rate of $500 \mathrm{~Hz}$.

\subsubsection{Computer task}

A gambling task was administered using Presentation software, version 13.0 (Neurobehavioral Systems, Inc., USA) (Figure 1). The task was modelled on games commonly run on EGMs. The screen display comprised a single row of four fruit symbols. Because eight different fruit symbols were used, and each could appear at random in each of the four columns, each trial was unique in terms of its stimulus configuration (symbol and sequence). After each trial, the amount wagered on that trial, the number of credits left to play with, and the amount won (if anything) were prominently displayed, as is typical of EGM displays. On each trial, one of four outcomes were possible: four identical symbols constituted a Large win, yielding ten times the amount bet; Small wins were indicated by three identical symbols that occurred in sequence, yielding five times the amount bet; Nearwins occurred when one different symbol was inserted between three identical symbols, and no credits were returned; and Losses occurred when neither a win nor near-win occurred, with no credits returned. The probability of the three main outcome types (wins, losses, and near-wins) closely matched outcomes from a real EGM (Wilkes et al., 2010). Although presented randomly, an equal number of Near-win (15\%) and Win (Small win: 7.5\%; Large win: $7.5 \%$ ) outcomes were presented, with the remaining trials being Losses (70\%). The current task differed from commercially available EGMs in that the visual and auditory 
stimuli that typically accompany EGM play were eliminated, so that ERPs to gambling outcomes were not confounded by these factors.

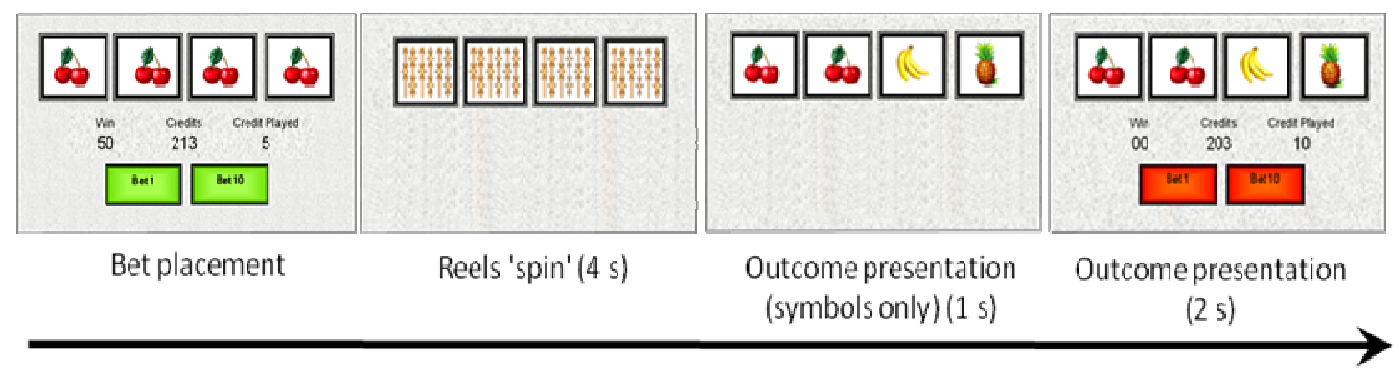

Figure 6. The sequence of events in the computer gambling task.

Participants did not wager their own money, but started the session with a free allocation of 5000 credits (valued at AUD50). Each trial commenced with participants choosing to either 'Bet Low' or 'Bet High' by pressing either a 'Bet 1' or 'Bet 10' button, respectively. Following this, a long inter-stimulus interval of $4 \mathrm{~s}( \pm 400 \mathrm{~ms})$, where the reels appeared to spin, was inserted between bet placement and the outcome (all reels stopped 'spinning' simultaneously) ensured that the epoch of interest was not contaminated by EEG activity associated with the immediately preceding event (i.e., activity related to the bet). Following the outcome presentation, a delay of $2 \mathrm{~s}$ occurred before the next bet could be placed (signalled by the 'Bet' button turning from red to green).

\subsection{Procedure}

After providing informed consent, participants anonymously completed the Canadian Problem Gambling Index (CPGI, Ferris and Wynne, 2001), which was used as a screening tool to assess diagnostic status and severity of gambling behaviours (individuals who score eight or above are classified as problem gamblers); no participants in the current study met the criteria for problem gambling. They also completed questionnaires that assessed their levels of reward and punishment sensitivity, gambling-related cognitions, levels of depression 
and anxiety, and impulsivity; due to low variability in these scores, the results of these inventories will not be reported here. Participants were then fitted with the physiological recording equipment and instructed on how to play the gambling task.

Eye movement data were obtained using an eye calibration task (Croft and Barry, 2000) that allows the offline correction of eye artifacts in task-related brain activity. After engaging in ten practice trials, participants played 450 trials on the gambling task. They were informed they would commence the task with 5000 credits and that they would win one entertainment voucher (valued at AUD12) if they had accumulated more than 6000 credits at the end of the experiment, and two vouchers if they had accumulated 7000 or more credits. Participants completed a subjective experience questionnaire immediately after the gambling task that assessed their levels of task related enjoyment and excitement (e.g., 'How excited did you feel when you experienced a Large win/Small win/Near-win/Loss while playing the poker machine', with answers ranging from $1=$ not excited to $9=$ very excited).

\subsection{Data reduction and analysis}

Ocular artifacts were removed using an eye-movement correction algorithm (Croft and Barry, 2000). EEG data were low-pass filtered below $30 \mathrm{~Hz}(24 \mathrm{~dB})$ and baseline corrected relative to the pre-stimulus interval $(100 \mathrm{~ms})$. Trials that contained muscle or other artifact were manually identified and excluded from further analysis. Once all artifacts were removed from the data, ERPs ranging from $100 \mathrm{~ms}$ to $800 \mathrm{~ms}$ post-stimulus were created for each participant over 19 sites (FP1, FP2, F3, F7 Fz, F4, F8, C3, C7, Cz, C4, C8, P3, P7, Pz, $\mathrm{P} 4, \mathrm{P} 8, \mathrm{O} 1, \mathrm{O} 2)$. Because there were not enough epochs for small and large wins over the two bet options for an examination of Outcome $\times$ Bet size interaction effects (see Results section 3.1), and because preliminary analyses showed the effect of bet size was small (see Figure 2A for effects at $\mathrm{Pz}$ and Figure 4A for effects at $\mathrm{Fz}$ ), we averaged the data from the Bet Low and 
Bet High epochs together to derive four Outcome types (Large wins, Small wins, Losses, Near wins). Furthermore, to prevent possible effects of habituation on the feedback related response (FRR) component/s, only the first $15 \%$ of Loss outcomes were included in the analyses.

In order to examine the latent ERP components associated with incentive value processing, a two-step spatiotemporal PCA was conducted on the data using the ERP PCA (EP) Toolkit, version 2.23 (Dien, 2010b). This data reduction technique was chosen over a temporospatial PCA, as the topographies of the ERP components of interest are wellestablished in the literature, and we wished to minimise the possibility of excluding ERP components with similar latencies but different topographies. A spatial PCA was first conducted on the data in order to identify the variance accounted for by electrode sites over all time points, participants and conditions. A temporal PCA, which uses all time points over all participants and conditions as variables, was then performed on the spatial components. The covariance matrix was used for all PCAs, with Kaiser normalisation. In accordance with the recommendation of Dien (2010a), INFOMAX rotation was performed for the spatial step of the PCA, and PROMAX rotation was performed for the temporal step of the PCA.

Once ERP components relevant to the paradigm used were identified, the data for each component were submitted to a four Outcome (Large win, Small win, Loss, Near-win) repeated measures analysis of variance (ANOVA). Orthogonal planned contrasts were conducted to examine differences between Wins (Large and Small combined) and Losses (Losses and Near-wins combined), Large wins and Small wins, and between Losses and Near-wins.

\section{Results}

\subsection{Behavioural data and reports of subjective experience}


On average, participants selected the Bet Low option on $38.9 \%$ of trials. Eight of the seventeen participants chose to Bet High at least $75 \%$ of the time, with seven of the total number of datasets containing five or fewer epochs in at least one of the win conditions (Bet Low/Small win, Bet Low/Large win, Bet High/Small win, Bet High/Large win), and fifteen of the total sample containing ten or fewer epochs (thus, as previously mentioned, outcomes were collapsed across the two bet options for analysis).

A repeated measures ANOVA revealed participants rated Large wins $(M=5.47, S D=$ $1.91)$ as more exciting than Small wins $(M=3.59, S D=1.46), F(1,16)=48.76, p<.001, \eta_{\mathrm{p}}^{2}$ $=.75$, and Small wins as more exciting as Losses $(M=2.35, S D=1.45), F(1,16)=8.25, p=$ $\left..011, \eta_{\mathrm{p}}^{2}=.34\right)$

\subsection{Physiological measures}

Dien (2010b; 2012) currently recommends using the parallel test (Horn, 1965), which compares the amount of variability explained in the observed dataset using the Scree test (Cattell, 1966) to that derived from uncorrelated variables within a dataset of totally random noise, to determine the number of factors to retain in the first step of a PCA. When applying this criterion, two site groupings (parietal and central, centred on $\mathrm{Pz}$ and Fz, respectively) were identified to account for the majority of variance in the dataset

The minimum percentage criterion calculated by the EP Toolkit was used to determine the number of temporal factors to be included in the second step of the PCA, as the parallel test recommended only four factors to be included, and none of these corresponded to the FRR ERP component/s. According to this criterion, seven temporal factors were found to best represent the data.

Parietal factor SF1/TF1, and frontal factor SF2/TF3, most closely corresponded to the P3b and FRN, respectively, in terms of their topography, latency, and response to 
experimental manipulations. Two of the six remaining parietal factors (positive Slow wave and Error positivity), and three of the six remaining frontal factors (negative Slow wave, and P200), were identifiable ERP components; however, these either did not show factor score differences between wins and losses, are of less theoretical importance, and/or are unrelated to the objectives of the current paper, so will not be discussed further.

\subsection{1. $P 300$}

Although both win and loss outcome types appeared to differ according to bet size (Figure 2A), preliminary Outcome $\times$ Bet analyses showed these effects were not significant. Thus, as mentioned above, because there were an insufficient number of epochs for a comparison of small and large wins following decisions to Bet Low and to Bet High, the PCA was conducted on data collapsed across the two Bet options.

The grand average virtual ERPs created by the EP toolkit were found to closely resemble the grand average raw ERP waveforms for this dataset at site Pz (SF1) (Figure 2B). Figure $2 \mathrm{C}$ shows how the grand average virtual ERP is comprised of the individual frontal factors that were identifiable ERP components, and/or accounted for at least $1 \%$ of the variance in the data. 


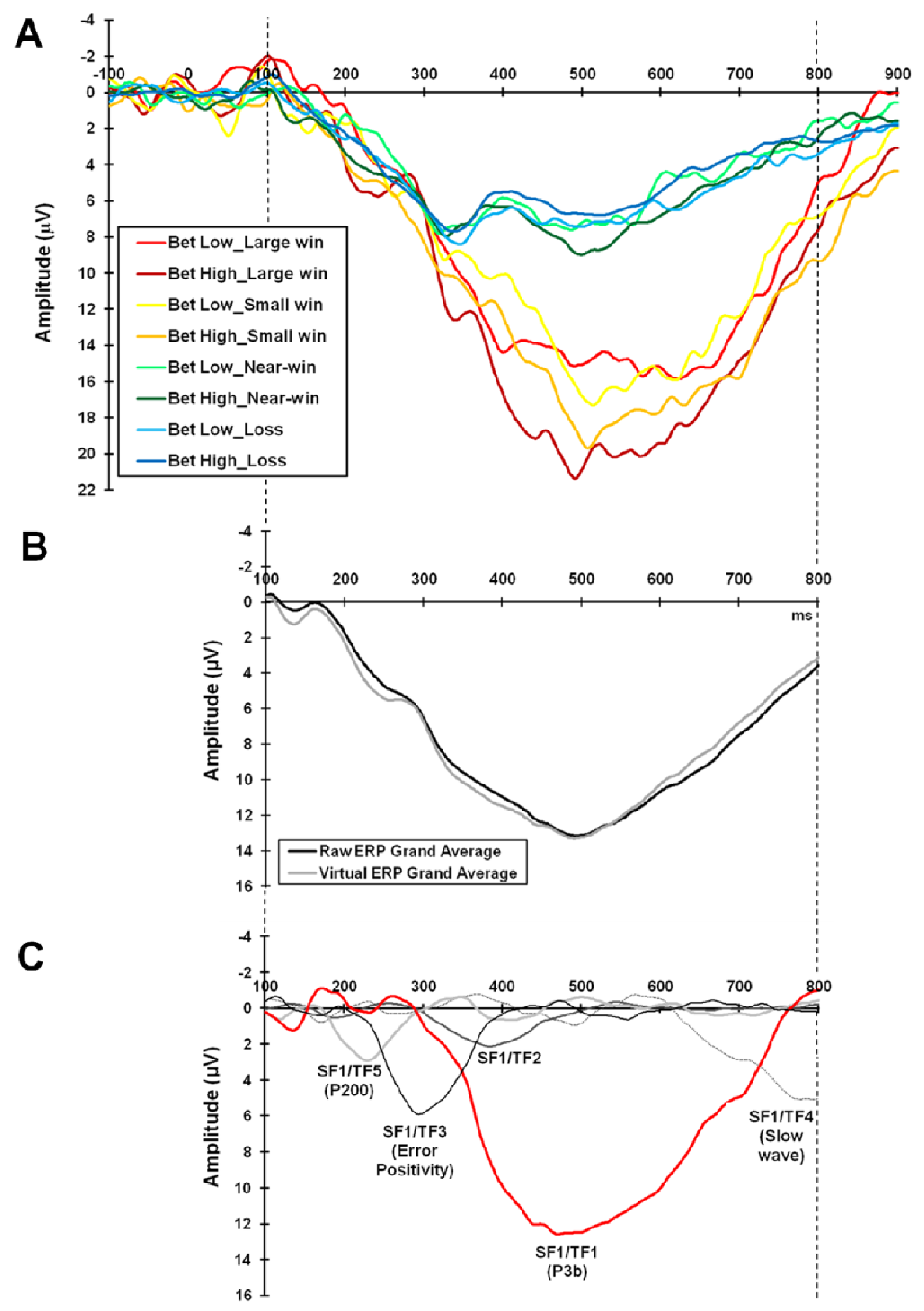

Figure 2. Waveform data corresponding to the parietally maximal ERP components (SF1). A) The raw ERP waveforms based on outcomes for each bet size at site Pz. B) The raw ERP grand average and virtual ERP grand average (over all outcomes and bet types) at site Pz, where the effect of the P3b was found to be greatest. C) The virtual ERP components averaged over all outcomes at site Pz for the five individual temporal factors that were identifiable ERP components, and/or accounted for more than $1 \%$ of the variance in the dataset. 
The factor that corresponded to the P3b ERP component (SF1/TF1) showed significantly larger amplitudes for Wins compared to Losses, $472 \mathrm{~ms}$ post-outcome, $F(1,16)$ $=61.76, p<.001, \eta_{\mathrm{p}}^{2}=.79$. Amplitudes did not differ between Small and Large wins, or between Losses and Near-wins (Figure 3).

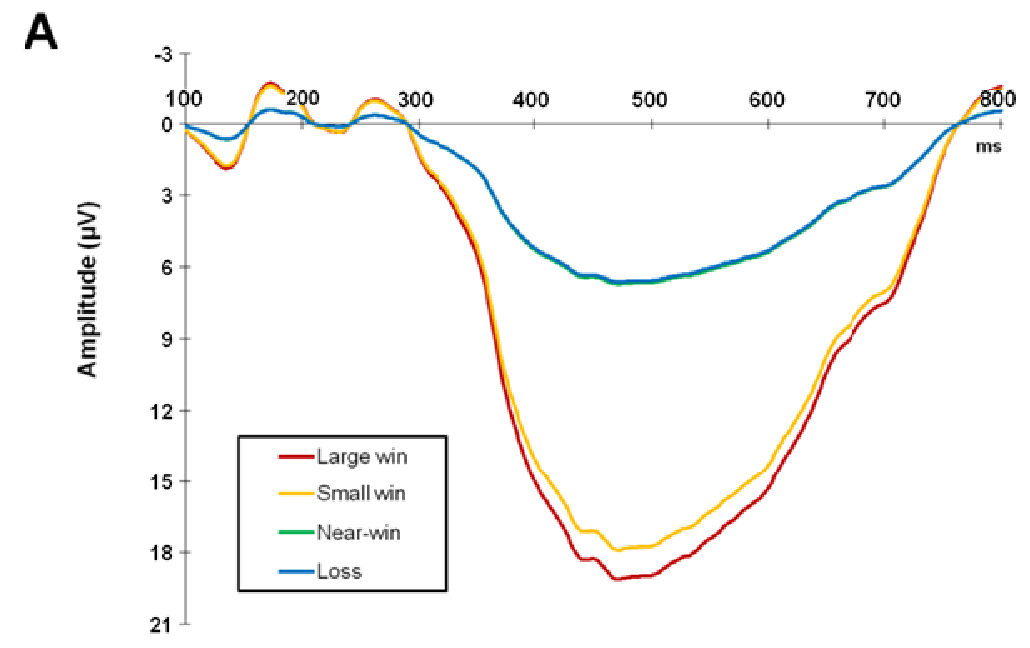

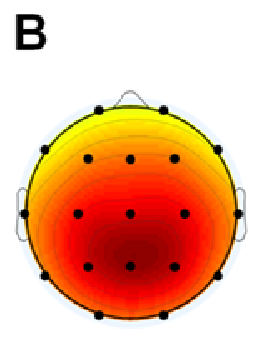

Large win

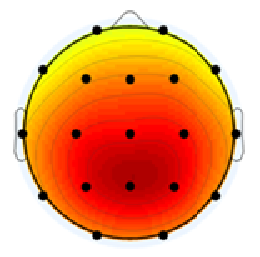

Small win

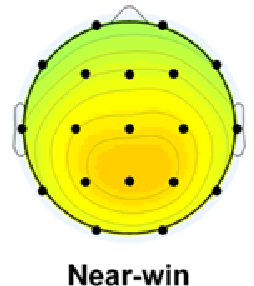

Near-win

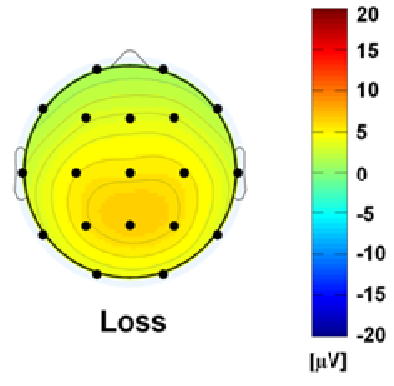

Figure 3. A) Virtual ERP waveforms for each outcome type for spatiotemporal factor, SF1/TF1, at site Pz. This factor was identified as corresponding to the P3b ERP component. B) Scalp topographies demonstrating the difference between win types, near-wins, and losses $472 \mathrm{~ms}$ post-outcome.

\subsubsection{Feedback related responses}

The raw waveforms for the average ERP responses following decisions to Bet High and to Bet Low for each Outcome type following can be seen in Figure 4A. The grand average virtual ERP was also found to closely resemble the grand average raw ERP 
waveforms for this dataset at site Fz (SF2) (Figure 4B). Figure 4C shows how the seven individual factors included in the PCA for this site constitute the grand average virtual ERP.

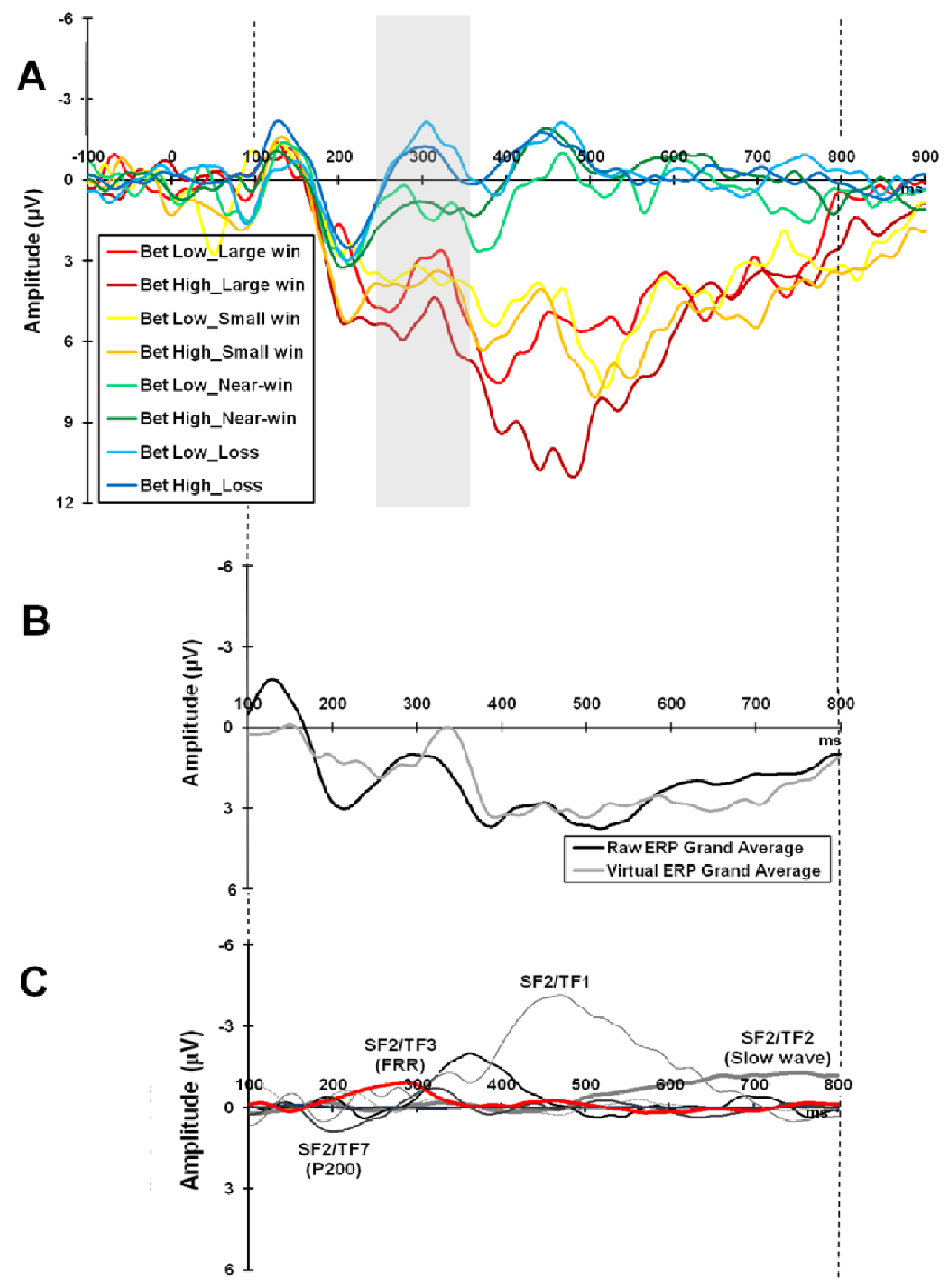

Figure 4.

Waveform data corresponding to the frontally maximal ERP components (SF2). A) The raw ERP waveforms based on outcomes for each bet size at site Fz. The highlighted section includes the peak that would have been considered to be the FRN using traditional (although non-difference waveform) quantification methods. B) The raw ERP grand average waveform and the virtual ERP grand average 
waveform (over all outcomes and bet sizes). C) The virtual ERP components averaged over all outcomes at site Fz for the four individual temporal factors that were identifiable ERP components, and/or accounted for more than $1 \%$ of the variance in the dataset.

The factor that most closely corresponded to the FRN ERP component (SF2/TF3) peaked at $290 \mathrm{~ms}$ post-outcome, and was characterised by both a negative deflection following Losses, and a positive deflection following Wins (Figure 5). The difference between win-loss outcomes was found to be statistically significant, $F(1,16)=126.85, p<$ $.001, \eta_{\mathrm{p}}{ }^{2}=.89$. Henceforth, negative deflections of this factor following losses will be referred to as FRN, and positive deflections to wins will be called feedback related positivity (FRP). Significantly larger FRP amplitudes were observed following Large wins compared to Small wins, $F(1,16)=7.21, p=.016, \eta_{\mathrm{p}}{ }^{2}=.31$. FRN amplitude following Losses were found to elicit significantly greater negative amplitudes compared to Near-wins, $F(1,16)=23.24$, $p$ $<.001, \eta_{\mathrm{p}}^{2}=.59$ 
A

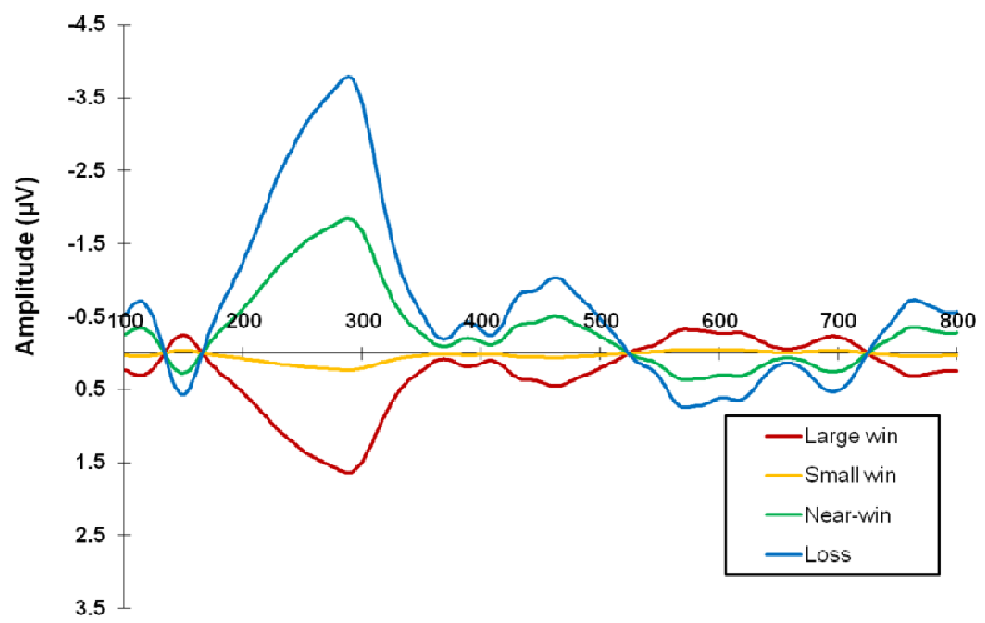

B

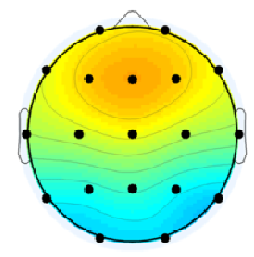

Large win

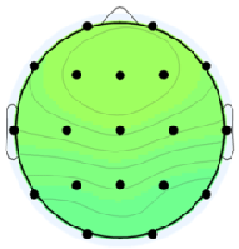

Small win

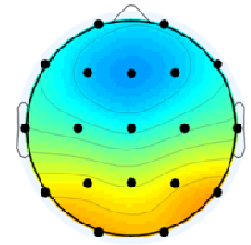

Near-win

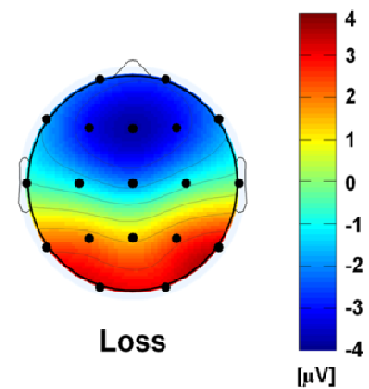

$[\mu \mathrm{V}]$

Figure 5. A) Virtual ERP waveforms for each outcome type for spatiotemporal factor, SF2/TF3, which was maximal at Fz. This factor was identified as corresponding to the feedback-related negativity and the feedback related positivity ERP components. B) Scalp topographies demonstrating the difference between wins, near-wins, and losses, $290 \mathrm{~ms}$ post-outcome.

\section{Discussion}

The current study used a PCA to investigate the latent nature of two ERP components previously found to index various aspects of incentive value processing. Specifically, we aimed to examine whether the feedback related responses (the FRN and the FRP) and the P3b could be considered distinct neural measures, and to determine whether they can be used as indices of the cognitive processing of outcome valence and reward magnitude in an ecologically valid paradigm. 


\subsection{Wins vs. losses}

\subsubsection{FRN/FRP}

By using a spatiotemporal PCA to parse the FRN from overlapping ERP components, we demonstrated that loss outcomes are reflected by a negative deflection approximately $290 \mathrm{~ms}$ post-feedback, a result consistent with previous FRN findings using traditional quantification methods (Gehring and Willoughby, 2002; Hajcak, et al., 2005; Hajcak, et al., 2006; Holroyd et al., 2006; Yeung, et al., 2005; Yeung and Sanfey, 2004). Conversely, we also demonstrated that win outcomes elicit a positive deflection at the same latency, corroborating the findings from other studies (Foti et al., 2011; Holroyd et al., 2003; Holroyd et al., 2008). The results of the current study serve to elucidate how feedback of differing incentive value are processed in the brain, as well as to consolidate and explain the disparate findings of the extant literature - something that is of major significance, as it affords the examination of the effects of both reward and punishment via independent neural correlates that are likely to be generated within the same cortical structure/s and/or system/s. The reinforcement learning theory (Holroyd and Coles, 2002), posits that outcomes are evaluated within the mesencephalic dopamine system based on previously learned expectations. Within this system, the basal ganglia monitor outcomes and stimulate dopamine levels: accordingly, rewarding outcomes cause dopamine to be released, leading to feelings of pleasure, whereas worse-than-expected outcomes are associated with reduced dopamine transmission (Fiorillo et al., 2003; Schultz, 2007). The results of the current study are compatible with this conjecture; the observed FRN deflection to negative feedback is likely to reflect the disinhibition of the ACC, whereas the FRP following reward outcomes reflects inhibition of the ACC - serving to guide future behaviours to aversive and appetitive stimuli, respectively.

Despite the probability of reward being controlled for in the analysis of the current study, the positive response to win outcomes observed may be exaggerated due to 
participants perceiving rewards to occur less frequently during the course of the experiment; however, a large positive deflection (i.e., the FRP) to reward outcomes, similar to that found in the current experiment, was also found in a recent study that used a temporospatial PCA to examine equiprobable win and loss outcomes in a simple two-choice gambling task (Foti et al., 2011). That study also found a much smaller FRN to loss outcomes, the reasons for which are unclear; such a result would not be predicted by stimulus frequency effects (losses occurred comparatively more frequently in the current study). It is possible that the more centrally maximal distribution of the component identified by Foti and colleagues may indicate a separate ERP component that is different from the feedback related responses of the current study, or may reflect the different paradigms used by each study; further research is required to consolidate these findings.

Indices of incentive value processing, including the FRN and FRP, are particularly relevant for the examination of individuals who have been posited to display abnormalities in the way rewarding and/or punishing/non-rewarding stimuli are evaluated. For example, attenuated or accentuated amplitudes of the positive deflection following wins in the response profile of problem gamblers may indicate a hyposensitivity or hypersensitivity to rewards, respectively, whereas reduced amplitudes of the negative deflection following losses may indicate a hyposensitivity to punishment. An examination of the responses to win and loss stimuli in such individuals may lead to the identification of a biological marker for addiction, and provide a foundation on which clinical treatments and interventions can be developed.

\subsection{2. $P 3 b$}

As predicted, we found larger P3b amplitudes for wins compared to losses, a result consistent with prior reports on global P300 amplitudes by some researchers (e.g., Hajcak et al., 2007), but not by others (Sato et al., 2005; Yeung and Sanfey, 2004). Thus, we did not corroborate the double-dissociation pattern suggested previously (Sato et al., 2005; Yeung 
and Sanfey, 2004), as both P3b and FRN were seen to be sensitive to valence. Although the probability of win (15\%), near-win (15\%), and loss events (70\%) were not matched in the EGM task of the current study, probability differences between these events fail to explain the differences observed. For instance, larger P3b amplitudes were observed to win events compared to equiprobable near-win events (see Figure 3). While it is theoretically possible that probability effects may have cancelled out an actual difference between near-wins and losses, this is unlikely because the two individual effects would be expected to work in the same direction: lower probability and great salience for near-wins would be expected to increase P3b effects. We conclude that the larger P3b amplitudes observed for wins (compared with losses) are associated with the motivational significance of rewards to the individual in response to ecologically valid stimuli, reflecting factors other than probability that are known to affect the P300 (Johnson, 1986).

\subsection{Effects of reward magnitude}

The current study added to previous research by examining whether reward magnitude affects FRP and P3b amplitude. The majority of previous studies have found that the traditionally conceptualised FRN is not sensitive to outcome magnitude (Hajcak et al., 2006; Sato et al., 2005; Yeung and Sanfey, 2004; Yu and Zhou, 2006; cf. San Martin et al., 2010), indicating that it reflects a dichotomous evaluation of outcomes, with events being contextually better or worse, with no response differences for intermediate-sized outcomes (Hajcak et al., 2006). The finding that the feedback related responses in the current study not only differentiates the valence of outcomes, but also that the FRP is sensitive to the magnitude of win outcomes is exciting, particularly for the future examination of problem gambling. It could help determine whether the behaviours that are characteristic of this disorder stem from abnormal responses to large and small wins; for example, an attenuated 
response to small, but not large wins, may suggest a predisposition to seek larger rewards despite personal cost.

Unlike the FRP, similar P3b component responses were found following small and large gains, a result that contrasts with earlier research (Sato et al., 2005; Yeung and Sanfey, 2004). It is unlikely that this result was due to the lack of difference in the perceived value of the amounts returned for large and small wins, as these amounts were comparable to those returned in previous studies where differences in P300 amplitude were observed for different magnitude wins (e.g., Bellbaum et al., 2010). Furthermore, although the subjective experience questionnaire did not specifically assess the perceived value of credits returned in the current task directly, participants did rate larger wins as more exciting than small wins.

\subsection{Near-wins}

While P3b amplitudes following near-wins suggest they are not as rewarding as wins (Figure 3), these outcomes were associated with reduced FRN amplitudes (Figure 5), implying that they are subjectively experienced as less aversive than full-loss outcomes, despite returning nil credits. These results are consistent with previous ERP research (Luo et al., 2011; cf. Qi et al., 2011), where near-wins resulted in reduced FRN, but no difference in P300 amplitudes, and suggest that these frequently occurring outcomes, that are unique to EGM gambling, are an important design feature of this gambling medium and may play a role in the development and maintenance of gambling behaviours by reducing the unpleasant nature of loss outcomes.

\subsection{Limitations and future directions}

Although attempts were made to construct an ecologically valid task, with stimulus characteristics, event-types and bet options simulating real gambling conditions, key differences between the laboratory task used in the current study and actual gambling need to be acknowledged. In real gambling, significant sums of money are wagered, won, and lost 
within a short period, whereas in previous laboratory-based studies (e.g., Lole et al., 2012; Wilkes et al., 2010), and the current study, rewards were capped and restricted to small amounts (movie voucher/s in the current study). Further, unlike real gambling, the participant does not suffer the risk of losing their own money, with losses often restricted to loss of free credits allocated to the research participant. It is often assumed that findings from controlled laboratory conditions will be replicated in, and may be generalised to actual gambling behaviors, but this remains to be demonstrated. Within the autonomic realm, research has examined electrodermal and cardiac data in real club settings when patrons bet with their own money (e.g., Coventry and Constable, 1999; Diskin and Hodgins, 2003; Griffiths, 1993; Krueger et al., 2005; Meyer et al., 2000). In a similar context, the implications of ERP findings for problem gambling have been alluded to in most laboratory studies, although we found no more than two studies that compared ERPs in response to reward and punishment sensitivity between problem and non-problem gamblers (Hewig et al., 2010; Oberg et al., 2011). The application of such information in the applied domain is clearly warranted and an urgent priority, although ethical and technical issues (e.g., challenges of recording reliable ERPs in a live-gambling environment) remain major challenges. Nevertheless, laboratory studies, where variables may be manipulated without the confounding factors that are encountered in vivo (e.g., the presence of others, financial implications, ingestion of psychoactive substances), are essential to establish a foundation of knowledge on which future applied research can build.

\subsection{Conclusions}

The application of a spatiotemporal PCA in the current study allowed an examination of the feedback related responses and P3b without the confounding influence of overlapping ERP components. The results of the feedback related responses from the current study consolidate those from previous research, in that two distinct ERP components sensitive to 
outcome valence occur at the same latency post-feedback; the FRN, characterised by a negative deflection to losses, and the FRP, characterised by a positive deflection to wins. The P3b was also confirmed to be a reliable index of reward valence, with greater amplitudes following win compared to loss outcomes. The current study was the first to examine the latent nature of the responses to different sized wins, and found the FRP, but not the P3b, to be a reliable index of reward magnitude, with greater amplitudes following large compared to small wins. The current study was also the first to examine near-wins using a PCA; these outcomes were found to elicit smaller FRN amplitudes compared to losses, which implies that they are perceived as less unfavourable than loss outcomes, and that they may play a role in the particular appeal of EGM gambling. Thus, the current study was able to achieve a comprehensive examination of the latent psychophysiological profile of healthy controls in response to gambling stimuli that are commonly encountered in real gambling environments. These findings may guide future research on problem gambling behaviours in order to determine whether this disorder is caused by an abnormal response to reward and/or punishment/non-reward stimuli.

\section{Acknowledgement}

This project was supported by an Australian Research Council Linkage Grant, LP0776836. 


\section{References}

Bellebaum, C., Daum, I., 2008. Learning-related changes in reward expectancy are reflected in the feedback-related negativity. Eur. J. Neurosci. 27, 1823-1835.

Bellbaum, C., Polezzi, D., Daum, I., 2010. It is less than you expected: The feedback-related negativity reflects violations of reward magnitude expectations. Neuropsychologia 48, 3343-3350.

Blum, K., Braverman, E.R., Holder, J.M., Lubar, J.F., Monastra, V.J., Miller, D. et al., 2000. Reward deficiency syndrome: A biogenetic model for the diagnosis and treatment of impulsive, addictive, and compulsive behaviors. J Psychoactive Drugs 32, 1-112.

Breen, R.B., Zimmerman, M., 2002. Rapid onset of pathological gambling in machine gamblers. J. Gambl. Stud. 18, 31-43.

Cattell, R.B., 1966. The scree test for the number of factors. Multivariate Behav. Res. 1, 245-276.

Clark, L., Lawrence, A.J., Astley-Jones, F., Gray, N., 2009. Gambling near-misses enhance motivation to gamble and recruit win-related brain circuitry. Neuron 61, 481-490.

Coventry, K.R., Constable, B., 1999. Physiological arousal and sensation-seeking in female fruit machine gamblers. Addiction 94, 425-430.

Croft, R.J., Barry, R.J., 2000. EOG correction of blinks with saccade coefficients: A test and revision of the aligned-artifact average solution. Clin. Neurophysiol. 3, 444-455.

De Pascalis, V., Varriale, V., D'Antuono, L., 2010. Event-related components of the punishment and reward sensitivity. Clin. Neurophysiol. 121, 60-76. 
Dien, J., 2010a. Evaluating two-step PCA of ERP data with Geomin, Infomax, Oblimin, Promax, and Varimax rotations. Psychophysiology 47, 170-183

Dien, J., 2010b. The ERP PCA Toolkit: An open source program for advanced statistical analysis of event-related potential data. J. Neurosci. Meth. 187, 138-145.

Dien, J., 2012. Applying principal components analysis to event-related potentials: A tutorial. Dev. Neuropsychol. 37, 497-517.

Diskin, K.M., Hodgins, D.C., 2003. Psychophysiological and subjective arousal during gambling in pathological and non-pathological video lottery gamblers. International Gambling Studies 3, 37-51.

Donchin, E., Coles, M.G., 1988. Is the P300 component a manifestation of context updating? Behav. Brain Sci. 11, 355-372.

Dowling, N., Smith, D., Thomas, T., 2001. Electronic gaming machines: Are they the 'crackcocaine' of gambling? Addiction 100, 33-45.

Dunning, J.P., Hajcak, G., 2007. Error-related negativities elicited by monetary loss and cues that predict loss. NeuroReport 18, 1875-1878.

Ferris, J., Wynne, H., 2001. The Canadian Problem Gambling Index: Final Report. Ottawa: Canadian Centre on Substance Abuse.

Fiorillo, C.D., Tobler, P.N., Schultz, W., 2003. Discrete coding of reward probability and uncertainty by dopamine neurons. Science 299, $1898-1902$.

Foti, D., Hajcak, G., 2009. Depression and reduced sensitivity to non-rewards versus rewards: Evidence from event-related potentials. Biol. Psychol. 81, 1-8.

Foti, D., Weinberg, A., Dien, J., Hajcak, G., 2011. Event-related potential activity in the basal ganglia differentiates rewards from nonrewards: Temporospatial principal 
components analysis and source localization of the feedback negativity. Hum. Brain Mapp. 32, 2207-2216.

Gehring, W.J., Willoughby, A.R., 2002. The medial frontal cortex and the rapid processing of monetary gains and losses. Science 295, 2279-2282.

Gonsalvez, C.J., Barry, R.J., Rushby, J., Polich, J., 2007. Target-to-target interval, intensity and P300 from an auditory single-stimulus task. Psychophysiology 44, 245-250.

Griffiths, M., 1993. Tolerance in gambling: An objective measure using the psychophysiological analysis of male fruit machine gamblers. Addict. Behav. 18, 365372.

Gu, R., Huang, Y., Luo, Y., 2010. Anxiety and feedback negativity. Psychophysiology 47, 961-967.

Hajcak, G., Holroyd, C.B., Moser, J.S., Simons, R.F., 2005. Brain potentials associated with expected and unexpected good and bad outcomes. Psychophysiology 42, 161-170.

Hajcak, G., Moser, J.S., Holroyd, C.B., Simons, R.F., 2006. The feedback-related negativity reflects the binary evaluation of good versus bad outcomes. Biol. Psychol. 71, 148154.

Hajcak, G., Moser, J.S., Holroyd, C.B., Simons, R.F., 2007. It's worse than you thought: The feedback negativity and violations of reward prediction in gambling tasks. Psychophysiology 44, 905-912.

Hewig, J., Trippe, R.H., Hecht, H., Coles, M.G.H., Holroyd, C.B., Miltner, W.H.R., 2007. Decision-making in blackjack: An electrophysiological analysis. Cerebr. Cortex 17, 865-877. 
Hewig, J., Kretschmer, N., Trippe, R.H., Hecht, H., Coles, M.G.H., Holroyd, C.B., Miltner, W.H.R., 2010. Hypersensitivity to reward in problem gamblers. Biol. Psychiatr. 67, 781-783.

Holroyd, C.B., Coles, M.G., 2002. The neural basis of human error processing: Reinforcement learning, dopamine, and the error-related negativity. Psychol. Rev. 109, 679-709.

Holroyd, C.B., Hajcak, G., Larsen, J.T., 2006. The good, the bad and the neutral: Electrophysiological responses to feedback stimuli. Brain Res. 1105, 93-101.

Holroyd, C.B., Krigolsen, O.E., 2007. Reward prediction error signals associated with a modified time estimation task. Psychophysiology 44, 913-917.

Holroyd, C.B., Larsen, J.T., Cohen, J.D., 2004. Context dependence of the event-related brain potential associated with reward and punishment. Psychophysiology 41, 245-253.

Holroyd, C.B., Niewenhuis, S., Yeung, N., Cohen, J.D., 2003. Errors in reward prediction are reflected in the event-related brain potential. NeuroReport 14, 2481-2484.

Holroyd, C.B., Pakzad-Vaezi, K.L., Krigolson, O.E., 2008. The feedback correct-related positivity: Sensitivity of the event-related brain potential to unexpected positive feedback. Psychophysiology 45, 688-697.

Horn, J.L., 1965. A rationale and test for the number of factors in factor analysis. Psychometrika 30, 179-185.

Johnson, R., Jr., 1986. A triarchic model of P300 amplitude. Psychophysiology 23, 367-384.

Krueger, T.H.C., Schedlowski, M., Meyer, G., 2005. Cortisol and heart rate measures during casion gambling in relation to impulsivity. Neuropsychobiology 52, 206-211. 
Lole, L., Gonsalvez, C.J., Blaszczynski, A., Clarke, A.R., 2012. Electrodermal activity reliably captures physiological differences between wins and losses during gambling on electronic machines. Psychophysiology 49, 154-163.

Luo, Q., Wang, Y., Qu, C., 2011. The near-miss effect in slot-machine gambling: Modulation of feedback-related negativity by subjective value. Neuroreport 22, 989-993.

Meyer, G., Hauffa, B.P., Schedlowski, M., Pawlak, C., Stadler, M.A., Exton, M.S., 2000. Casino gambling increases heart rate and salivary cortisol in regular gamblers. Biol. Psychiatr. 48, 948-953.

Miltner, W.H.R., Braun, C.H., Coles, M.G.H., 1997. Event-related brain potentials following incorrect feedback in a time-estimation task: Evidence for a "generic" neural system for error detection. J. Cogn. Neurosci. 9, 788-798.

Nieuwenhuis, S., Slagter, H.A., von Geusau, N.J.A., Heslenfeld, D., Holroyd, C.B., 2005. Knowing good from bad: Differential activation of human cortical areas by positive and negative outcomes. Eur. J. Neurosci. 21, 3161-3168.

Nieuwenhuis, S., Yeung, N., Holroyd, C.B., Schurger, A., Cohen, J.D., 2004. Sensitivity of electrophysiological activity from medial frontal cortex to utilitarian and performance feedback. Cereb. Cortex 14, 741-747.

Oberg, S.A.K., Christie, G.J., Tata, M.S., 2011. Problem gamblers exhibit reward hypersensitivity in medial frontal cortex during gambling. Neuropsychologia 49, $3768-3775$.

Petry, N.M., 2003. A comparison of treatment-seeking pathological gamblers based on preferred gambling activity. Addiction 98, 645-655.

Qi, S., Ding, C., Song, Y., Yang, D., 2011. Neural correlates of near-misses effect in gambling. Neurosci. Lett. 493, 80-85. 
Reuter, J., Raedler, T., Rose, M., Hand, I., Glascher, J., Buchel, C., 2005. Pathological gambling is linked to reduced activation of the mesolimbic reward system. Nat. Neurosci. 8,147-148.

San Martin, R., Manes, F., Hurtado, E., Isla, P., Ibanez, A., 2010. Size and probability of rewards modulate the feedback error-related negativity associated with wins but not losses in a monetarily rewarded gambling task. NeuroImage 51, 1194-1204.

Sato, A., Yasuda, A., Ohira, H., Miyawaki, K., Nishikawa, M., Kumano, H., Kuboki, T., 2005. Effects of value and reward magnitude on feedback negativity and P300. NeuroReport 16, 407-411.

Schultz, W., 2007. Behavioral dopamine signals. Trends Neurosci. 30, 203-210.

Toyomaki, A., Murohashi, H., 2005. The ERPs to feedback indicating monetary loss and gain on the game of modified "rock-paper-scissors". Int. Congr. 1278, 381-384.

Verleger, R., 1988. Event-related potentials and cognition: A critique of the context updating hypothesis and an alternative interpretation of P3. Behav. Brain Sci. 11, 343427.

Wilkes, B.L., Gonsalvez, C.J., Blaszczynski, A., 2010. Capturing SCL and HR changes to win and loss events during gambling on electronic machines. Int. J. Psychophysiol. $78,265-272$.

Wu, Y., Zhou, X., 2009. The P300 and reward valence, magnitude, and expectancy in outcome evaluation. Brain Res. 1286, 114-122.

Yeung, N., Holroyd, C.B., Cohen, J.D., 2005. ERP correlates of feedback and reward processing in the presence and absence of response choice. Cereb. Cortex 15, 535544.

Yeung, N., Sanfey, A.G., 2004. Independent coding of reward magnitude and valence in the human brain. J. Neurosci. 24, 6258-6264. 
Yu, R., Zhou, X., 2006. Brain responses to outcomes of one's own and other's performance in a gambling task. NeuroReport 17, 1747-1751.

Zhou, Z., Yu, R., Zhou, X., 2010. To do or not to do? Action enlarges the FRN and P300 effects in outcome evaluation. Neuropsychologia 48, 3606-3613. 


\section{Figure Captions}

Figure 1. The sequence of events in the computer gambling task.

Figure 2. Waveform data corresponding to the parietally maximal ERP components (SF1). A) The raw ERP waveforms based on outcomes for each bet size at site Pz. B) The raw ERP grand average and virtual ERP grand average (over all outcomes and bet types) at site Pz, where the effect of the P3b was found to be greatest. C) The virtual ERP components averaged over all outcomes at site Pz for the five individual temporal factors that were identifiable ERP components, and/or accounted for more than $1 \%$ of the variance in the dataset.

Figure 3. A) Virtual ERP waveforms for each outcome type for spatiotemporal factor, SF1/TF1, at site Pz. This factor was identified as corresponding to the P3b ERP component. B) Scalp topographies demonstrating the difference between win types, near-wins, and losses $472 \mathrm{~ms}$ post-outcome.

Figure 4. Waveform data corresponding to the frontally maximal ERP components (SF2). A) The raw ERP waveforms based on outcomes for each bet size at site Fz. The highlighted section includes the peak that would have been considered to be the FRN using traditional (although non-difference waveform) quantification methods. B) The raw ERP grand average waveform and the virtual ERP grand average waveform (over all outcomes and bet sizes). C) The virtual ERP components averaged over all outcomes at site Fz for the four individual temporal factors that were identifiable ERP components, and/or accounted for more than $1 \%$ of the variance in the dataset.

Figure 5. A) Virtual ERP waveforms for each outcome type for spatiotemporal factor, SF2/TF3, which was maximal at Fz. This factor was identified as corresponding to the feedback-related negativity and the feedback related positivity ERP components. B) Scalp topographies demonstrating the difference between wins, near-wins, and losses, $290 \mathrm{~ms}$ post-outcome. 